Atmos. Chem. Phys. Discuss., 10, 7495-7506, 2010

www.atmos-chem-phys-discuss.net/10/7495/2010/

(C) Author(s) 2010. This work is distributed under

the Creative Commons Attribution 3.0 License.

This discussion paper is/has been under review for the journal Atmospheric Chemistry and Physics (ACP). Please refer to the corresponding final paper in ACP if available.

\title{
Why anisotropic turbulence matters: another reply
}

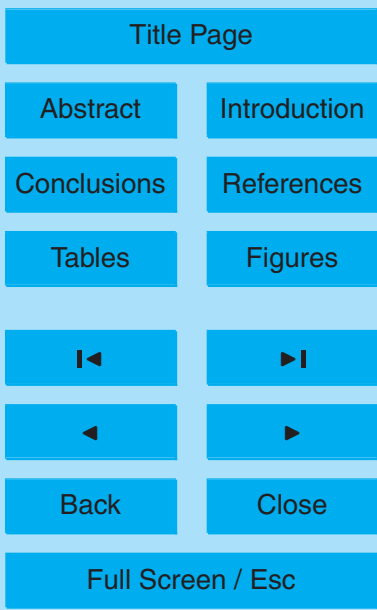

S. Lovejoy ${ }^{1{ }^{\star \star}}$, D. Schertzer ${ }^{2,3}$, and A. F. Tuck

${ }^{1}$ Physics, McGill University, 3600 University st., Montreal, Que. H3A 2T8, Canada

${ }^{2}$ CEREVE, Université Paris Est, France

${ }^{3}$ Météo France, 1 Quai Branly, Paris 75005, France

*currently at: Physics Department, Imperial College, London, UK

** on leave to: NOAA Earth System Research Laboratory, Physical Sciences Division, 325

Broadway, Boulder CO 80305-3337, USA

Received: 2 February 2010 - Accepted: 16 March 2010 - Published: 23 March 2010

Full Screen / Esc

Correspondence to: S. Lovejoy (lovejoy@ physics.mcgill.ca)

Published by Copernicus Publications on behalf of the European Geosciences Union.

Printer-friendly Version

Interactive Discussion 


\section{Abstract}

We continue the debate on anisotropic but scaling turbulence and its effect on aircraft measurements of turbulence (cf. Lindborg et al., 2010a, b); hereafter LTNCG1, LTNCG2). We revisit the repeatedly presented back-of-the-envelope calculation and

5 discuss wind statistics on real isobars. We then discuss theoretical and empirical evidence that a $k^{-5 / 3}$ horizontal wind spectrum could extend out to planetary scales.

\section{Introduction}

The reactions to our paper (Lovejoy et al., 2009), hereafter denoted LTSH) and to our initial responses (Lovejoy, 2009; Schertzer, 2009); (hereafter LTSH reply 1 and 10 LTSH reply 2 respectively) failed to address the relevant issue of anisotropic scaling turbulence and its effect on aircraft measurements. We therefore attempt to succinctly summarize the debate and then to revisit the substantive part in a somewhat different, hopefully more accessible manner.

\section{Scaling Isotropic versus scaling anistropic turbulence: the debate}

\subsection{Discussion}

Over the last twenty-five years there have been two competing statistical turbulent frameworks for atmospheric dynamics the "isotropy primary" (IP) "standard model" which postulates first isotropy and only then scaling and the "scaling primary" (SP) model. While the former implies at least two regimes separated by a "dimensional transition": a small scale regime of isotropic 3-D turbulence and a large scale regime of isotropic 2-D turbulence, the latter implies wide range scaling but with different exponents in the horizontal and vertical directions.

Why anisotropic turbulence matters: another reply

S. Lovejoy et al.

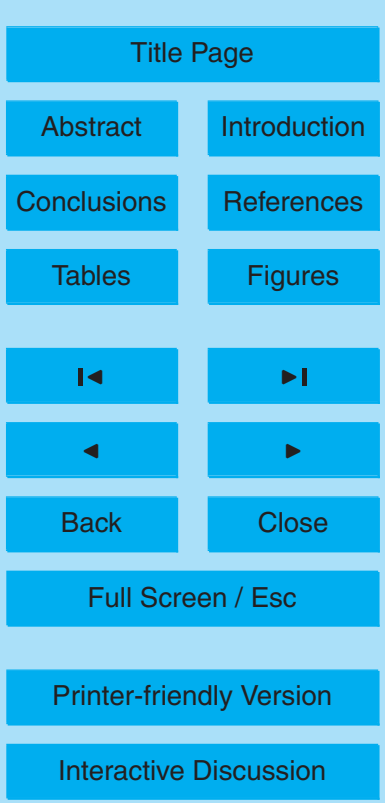


In our previous responses (and in publications cited therein), we already discussed this history and recent developments. Below we recall the salient criticisms of the IP approaches.

\subsection{Summary of critiques of isotropic turbulence based on the empirical evidence}

- A large body of evidence now exists showing that in the vertical atmospheric fields are accurately scaling but with exponents which are quite different than those in the horizontal and which are incompatible with isotropy.

- In the horizontal, satellite radiances - including the energy containing short and long wave radiances - demonstrate very accurate scaling over at least the range $10-5000 \mathrm{~km}$. Other in situ fields such as temperature and humidity are also accurately scaling through the meso-scale (see e.g. Fig. $3 e$ in LTSH). The wide range scaling of these fields is particularly significant since unlike the wind field which has a second quadratic invariant in 2-D (the enstrophy), these have only a single quadratic invariant in both 2-D and 3-D so that the postulated dimensional transition would - if indeed present - be particularly pronounced.

- The only field which systematically shows a break in the scaling is the horizontal wind field. However, the break occurs at a scale which is significantly larger than the scale thickness of the atmosphere (several hundred kilometres according to (Gage and Nastrom, 1986), and on average $\approx 40 \mathrm{~km}$ according to LTSH). In addition, while the large scale spectrum $(E(k))$ - is indeed of the scaling form $k^{-\beta}$ with a value $\beta$ significantly larger than $5 / 3$ - the actual value is not very close to the 2 -D turbulence value $\beta \approx 3$. In fact - as we have shown by detailed reviews virtually all the classical papers (including several reviewed in LTSH Sect. 5 which were apparently missed) - the value $\beta \approx 2.4$ predicted by the SP approaches is much closer to the data than $\beta \approx 3$. The statement in LTNCG2 that "the amount of

Why anisotropic turbulence matters: another reply

S. Lovejoy et al.

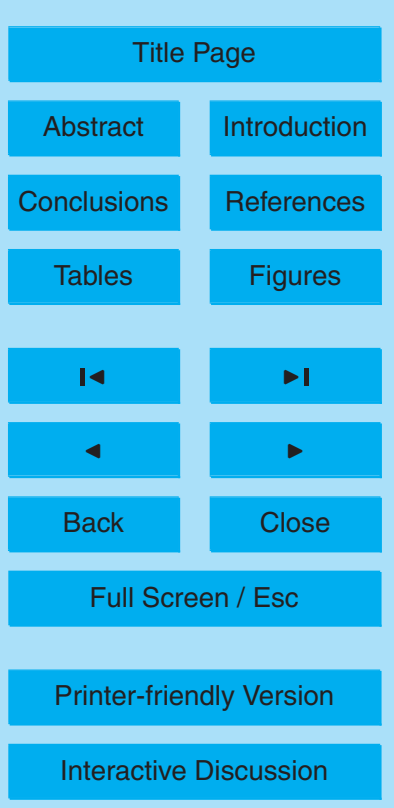

Interactive Discussion 
evidence in favour of an approximate $k^{-3}$ regime at synoptic scales is overwhelming" is only true if one only considers a priori that the only two values of $\beta$ which are physically possible are $5 / 3$ and 3 so that nearly exact $k^{-2.4}$ spectra can be considered "approximately" $k^{-3}$.

5 - Even if the horizontal wind apparently has a transition from $k^{-5 / 3}$ to $k^{-2.4}$ instead of from $k^{-5 / 3}$ to $k^{-3}$, it would still imply a break in the scaling which would at least restrict the range of validity of the SP approach. This is why it is so important to correctly understand the effect of anisotropic turbulence on the aircraft wind measurements - virtually our only source of information on the horizontal scaling properties of the wind. It is ironic that the LTSH developments that LTNCG2 term "irrelevant and obsolete" include an original explicit theoretical and empirical attempt to deduce the consequences of anisotropic turbulence on the aircraft and hence on the measurements. From this we can only surmise that the - presumably relevant and nonobsolete - isotropic turbulence framework in which the measurements have been exclusively interpreted for the last 40 years are considered satisfactory. In any case, an important consequence of this anisotropic measurement theory is that after some critical distance $\Delta x_{C}$, we expect the aircraft to begin to have statistics dominated by the vertical motions, and hence that we expect the spurious appearance of the (rough) vertical spectrum $k^{-2.4}$.

\subsection{Summary of the critique of isotropic turbulence based on the theoretical/numerical evidence}

1. The 2-D/3-D model was elaborated 40 years ago when scale invariance was still believed to be a necessarily isotropic symmetry. It has since emerged as an important new (nonclassical and anisotropic) symmetry of great generality, so that today it seems quite odd - if not perverse - to attempt to impose isotropy, especially on systems where there are several obvious sources of strong anisotropy gravity and the Coriolis force - to name but two.

Why anisotropic turbulence matters: another reply

S. Lovejoy et al.

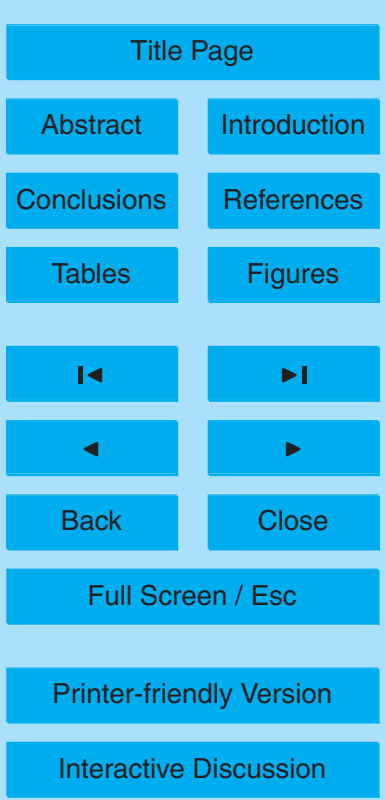


2. While the development of quasi-geostrophic turbulence (Charney, 1971) was historically important, it has many intrinsic limitations. Problematic assumptions and ingredients include: the existence of a scale separation between large and small scales, of hydrostatic equilibrium, of the use of the geostrophic wind to determine the material derivative, of the smallness of the Rossby number and of the near uniformity of the static stability (cf. LTSH reply 2 for details).

3. Over the last 25 years multiplicative cascade processes have also been greatly developed (see e.g. the overview Schertzer et al., 1997). They are now understood as the generic multifractal processes and are expected to generally apply in scale invariant systems having large numbers of degrees of freedom and in which a flux is conserved from scale to scale. This is much more general than the usual "inertial range" and it is therefore not surprising that it has recently been shown that several numerical weather models and several different reanalyses have nearly exact multiplicative cascade structures from about $5000 \mathrm{~km}$ down to their inner (hyper) viscous scales (Stolle et al., 2009).

4. There is growing evidence that any isotropic 3-D turbulence would destabilize ("three-dimensionalize") an isotropic 2-D regime so that the two would not be able to co-exist (Ngan et al., 2004). At least some of the computer simulations LTCNG1 cites as apparently producing both $k^{-5 / 3}$ and $k^{-3}$ regimes are in fact almost certainly displaying artefacts due to incorrect use of hyperviscous damping (as pointed out in Smith, 2004 and reiterated in LTSH reply 2), and in any case, most numerical models do not show the two regimes.

5. There is evidence from the ECMWF interim reanalysis that even isobaric crosssections of the zonal and meridional winds display anisotropic but scaling statistics with effective ("elliptical") dimensions of 1.80 rather than 2 . This is evidence that isotropy is strongly broken even on isobaric surfaces and makes the very special case of isotropic 2-D turbulence even less plausible (Lovejoy and Schertzer, 2010a).

Why anisotropic turbulence matters: another reply

S. Lovejoy et al.

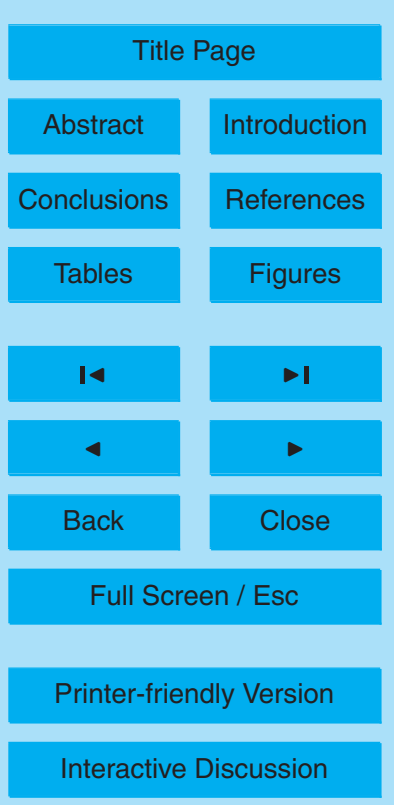

Interactive Discussion 


\section{The substantive comments}

\subsection{Revisiting the back-of-the-envelope}

LTNCG2 reiterates the back-of-the-envelope (LTNCG1) argument that "an inaccuracy or uncertainty of $100 \mathrm{~m}$ with respect to the vertical position of the aircraft cannot explain

5 a supposed wind shift of the order of $10 \mathrm{~m} / \mathrm{s}$, for the simple reason that the typical wind shift over a vertical distance of $100 \mathrm{~m}$ is less than $1 \mathrm{~m} / \mathrm{s}$." This argument is simplistic and simply ignores the detailed analyses presented in LTSH (the entirety of Sects. 2 and 3) which was already addressed at length in our previous response (LTSH reply, p C7695). The basic point is that if the ratio of horizontal to vertical exponents $\left(H_{z}\right)$ and sphero10 scale $\left(I_{\mathrm{s}}\right)$ is small enough and if the aircraft slopes $(s)$ are large enough, then there will be a critical transition scale $\Delta x_{\mathrm{c}}$ after which the aircraft will measure the vertical exponents rather than the horizontal ones. In the original paper we gave the theory and the equations and the parameter estimates and we showed that the numbers did indeed jibe very well with the average $\Delta x_{c}$ expected to be less than $1000 \mathrm{~km}$ (but with large flight to flight variations). By direct measurement, $\Delta x_{\mathrm{c}}$ was found to be a bit smaller than this simple model prediction; this was not surprising since as noted, the model ignores the statistical variability of the slopes and their correlations with the wind field (as predicted by approximate hydrostatic and geostrophic balances). Some of this complexity was glimpsed in the phase and coherence analysis between the wind and altitude and pressure in LTSH, but much work still needs to be done for it to be fully understood.

Since LTNCG2 reaffirmed the same argument as LTNCG1, our explanation in LTSH reply 1 and the arguments and data in the original LTSH paper were apparently not noticed. Let us therefore revisit this in an even simpler way by turning away from

LTNCG's hypothetical atmospheres and looking at the real world where isobars are not so simple. Each of the sixteen flights analysed in LTSH were isobaric to within $\pm 0.2 \mathrm{mb}$ yet the dynamic altitude range (the difference between the maximum and minimum altitude on the isobar) was frequently much greater than the cited $100 \mathrm{~m}$ value: in

Why anisotropic turbulence matters: another reply

S. Lovejoy et al.

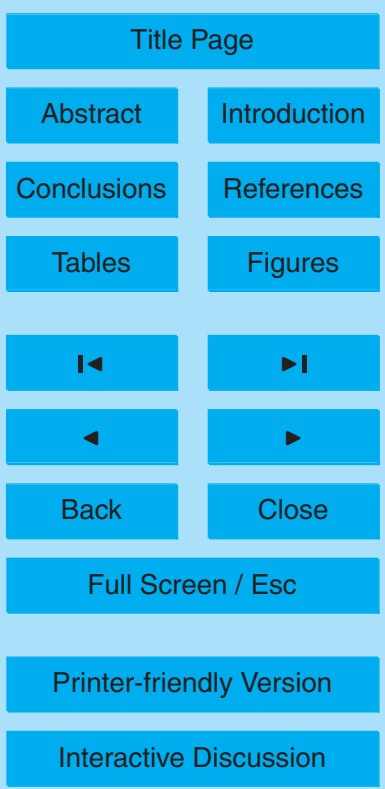


several cases it reached nearly $900 \mathrm{~m}$ on flight segments about $3000 \mathrm{~km}$ long; from Fig. $1 \mathrm{~b}$ in LTSH, it can be seen graphically that the difference can readily be $400 \mathrm{~m}$ over $1000 \mathrm{~km}$ sections (see also Table 1, LTSH for a summary). According to the analysis of data from drop-sondes dropped during the flights (Lovejoy et al., 2007), while one 5 would indeed expect on average a mean vector velocity difference of $\approx 1 \mathrm{~m} / \mathrm{s}$ over $100 \mathrm{~m}$ thick layers, over $400 \mathrm{~m}$ layers the corresponding value is closer to $2.8 \mathrm{~m} / \mathrm{s}$. But these values are averages over the entire troposphere, the corresponding mean drop-sonde value at the relevant aircraft height where vertical shear is quite pronounced ( $\approx 200 \mathrm{mb}$ ), is the much larger value $7.1 \mathrm{~m} / \mathrm{s}$ - and this average itself hides large variations (see

10 Fig. 1 for the full information; the Fig. $7.1 \mathrm{~m} / \mathrm{s}$ is obtained by extrapolating the $400 \mathrm{~m}$ curve to the mean aircraft altitude of $12.5 \mathrm{~km}$ ). Also indicated in Table 1 of LTSH are the mean horizontal velocity shears which imply that at separations of $1000 \mathrm{~km}$ mean horizontal velocity differences are frequently of the same order: for 11 of the 16 flights the difference was less than $10 \mathrm{~m} / \mathrm{s}$, the flight with the lowest mean difference being $154.9 \mathrm{~m} / \mathrm{s}$. Again these values are only the means. But even if we substitute these real values in place of LTNCG's hypothetical ones, it still only shows that the mechanism we propose is plausible: to go beyond this one must read Sects. 2-4 of LTSH.

\subsection{The isoheight spectra}

A more interesting affirmation is the statement "To our knowledge, there is not a sin20 gle piece of empirical evidence reported in the literature in favour of the hypothesis of Lovejoy et al. that the synoptic energy spectrum should scale as $k^{-5 / 3}$ ". This statement is contrary to both LTSH, the replies, and a number of other cited papers. The same is true of the next sentence: "Not even the measurements made by Lovejoy et al themselves support this hypothesis in any way". It seems that we must re-examine 25 this issue once more. It is at least scientifically more interesting because it is obvious that the finding that the large scale aircraft measurements spuriously yield the vertical exponent does not prove that the correct isoheight exponent is $\beta=5 / 3$. Let us therefore first resummarize the evidence given in the paper and in the previous response before

Why anisotropic turbulence matters: another reply

S. Lovejoy et al.

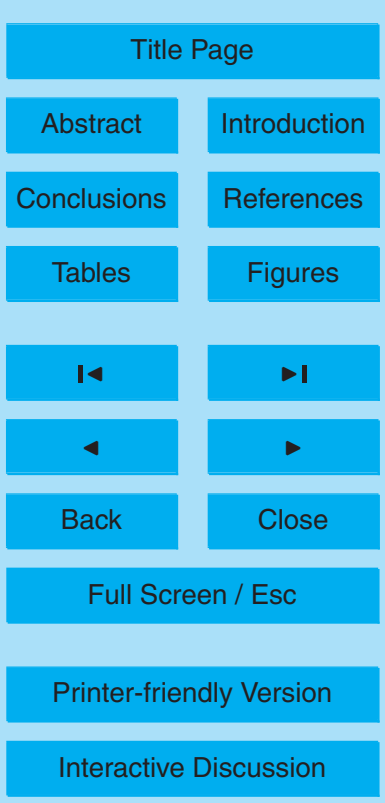


giving a somewhat new argument.

In the original LTSH paper the question about the true isoheight exponent was explicitly discussed - indeed an original data analysis method was developed to answer it (most of Sect. 4!). Unlike existing analysis techniques, this "scale invariant lag" method 5 simultaneously takes into account the vertical and horizontal fluctuations of the aircraft quantitatively distinguishing for example between short lags with large vertical excursions and long, flat lags. It showed agreement with the $\beta=5 / 3$ hypothesis over a stunning 8 orders of magnitude of the "scale invariant lag". In addition, it was noted that if there was indeed a break in the scaling of the spectrum at a scale of several 10 hundred kilometres (at seen in the Gage and Nastrom, 1986), then it would surely also be evident in satellite radiances or in situ temperature and humidity measurements (LTSH Fig. 3e). Finally, in our previous response (and in more detail in Lovejoy and Schertzer, 2010b) we already indicated that if the exponent $\beta=5 / 3$ holds out to planetary scales, then one can actually get an accurate estimate of the large scale wind gradients $(\approx 20 \mathrm{~m} / \mathrm{s})$ by using "first principles" starting with the solar constant then estimating the small scale energy flux and finally using the scaling to extrapolate this to planetary scales.

Let us put this argument a little differently. Consider the average difference in the horizontal wind across points $1 \mathrm{~km}$ distant; from Table 1 in LTSH (the column giving the small scale estimates of $\varepsilon$ ) we find that on average $\Delta v \approx 0.74 \mathrm{~m} / \mathrm{s}$. In comparison, using the ECMWF interim reanalysis for January 2006 , we find for $\Delta x \approx 330 \mathrm{~km}$, the mean velocity difference is $6.7 \mathrm{~m} / \mathrm{s}$ whereas for the antipodes $(\Delta x \approx 20000 \mathrm{~km})$, the corresponding value is $17.3 \mathrm{~m} / \mathrm{s}$ (Lovejoy and Schertzer, 2010b). If there is a unique scaling regime, then $\Delta v \propto \Delta x^{H}$ so that the exponent $H$ can be estimated by considering any 25 pair of values using $H=\log \left(\Delta v_{1} / \Delta v_{2}\right) / \log \left(\Delta x_{1} / \Delta x_{2}\right)$. Using the $1 \mathrm{~km}$ and $330 \mathrm{~km}$ pair we find $H \approx 0.38$ whereas using the $1 \mathrm{~km}$ and $20000 \mathrm{~km}$ pair, we find $H \approx 0.31$. Alternatively, we can use a regression through all three values and find $H \approx 0.32$. In all cases, the value $H=1 / 3$ (corresponding to $\beta=5 / 3$ without small intermittency corrections) does extraordinarily well. Indeed it is hard to see how there could be any significant large

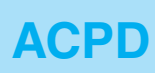

10, 7495-7506, 2010

\section{Why anisotropic turbulence matters: another reply}

S. Lovejoy et al.

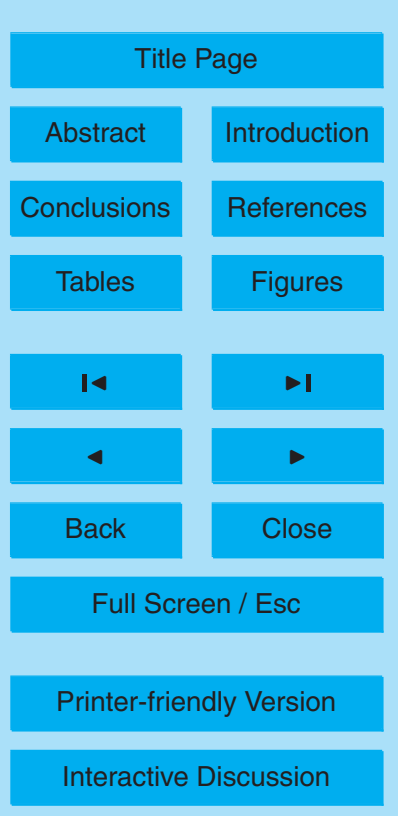


scale regime with either $\beta=2.4$ or $\beta=3$ isoheight exponents (corresponding to $H \approx 0.7$, 1). For example, even the existence of a range of factor of 10 in scale with $H=0.7$ $(\beta=2.4)$ instead of $1 / 3$ would give a value of the antipodes difference a factor 2.5 times too large; and using the $H=1(\beta=3)$ value for which LTCNG2 affirms there is "over5 whelming evidence" the difference is too big by a factor 4.6. In other words according to pretty basic data and being fairly generous (e.g. the values cited may not be representative enough of the ensemble statistics), it would be very difficult to hide a $\beta=3$ range covering more than a factor of about 3 in scale (which would already imply antipodes velocity differences a factor $\approx 2$ too large).

\section{Conclusions}

A simple theory of the effect of anisotropic turbulence on aircraft trajectories and the interpretation of turbulence measurements has only been available for a few years yet LTCNG2 considers it to be already "irrelevant" and "obsolete", apparently preferring that interpretations continue to be made using the familiar isotropic assumptions. On 15 the contrary, we believe that it is precisely the persistence of this outmoded isotropic paradigm which is preventing us from properly understanding both the measurements and the atmosphere's dynamics. While the anisotropic framework we propose gives a straightforward explanation of the classical aircraft measurement campaigns, there is still much work to be done if we are to fully understand the interaction of the aircraft's trajectories and the anisotropic turbulence that they measure. One example of this that we have already mentioned is the need to better understand the relation between aircraft slope and wind fluctuations, but there are other issues that deserve attention. For example, if the aircraft flies through a region with a varying vertical velocity, then the effect is exactly analogous to that of a constant slope and the consequences are the same; this effect will simply amplify the one already discussed; preliminary analysis shows that it is typically of comparable magnitude. Finally, a complication that we explore elsewhere (Lovejoy and Schertzer, 2010a) is the possibility (suggested by the

Why anisotropic turbulence matters: another reply

S. Lovejoy et al.

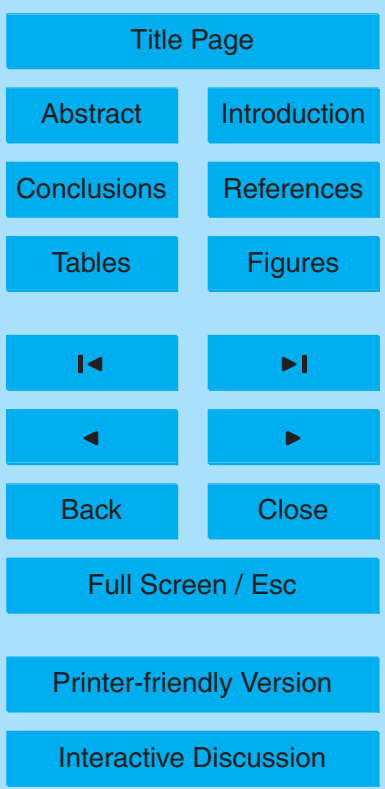

Interactive Discussion 
statistics of the zonal wind in the ECMWF reanalyses) that there is also a differential, scaling anisotropy in the horizontal between zonal and meridional directions. This implies that turbulent exponents are affected not only by vertical aircraft fluctuations, but also by the aircraft's heading: even in horizontal sections the turbulence appears to be 5 strongly anisotropic.

We hope that these additional comments and restatement of our earlier responses will clarify the important debate about the nature of atmospheric turbulence, dynamics by helping to eliminate "irrelevant" and "obsolete" ideas. This is necessary if a new nonlinear synthesis is to emerge capable of providing a unified modelling and empirical

\section{References}

Charney, J. G.: Geostrophic Turbulence, J. Atmos. Sci., 28, p. 1087, 1971.

Gage, K. S. and Nastrom, G. D.: Theoretical Interpretation of atmospheric wavenumber spectra of wind and temperature observed by commercial aircraft during GASP, J. Atmos. Sci., 43, 729-740, 1986.

Lindborg, E., Tung, K. K., Nastrom, G. D., Cho, J. Y. N., and Gage, K. S.: Comment on "Reinterpreting aircraft measurement in anisotropic scaling turbulence" by Lovejoy et al. (2009), Atmos. Chem. Phys., 10, 1401-1402, 2010, http://www.atmos-chem-phys.net/10/1401/2010/.

Lindborg, E., Tung, K. K., Nastrom, G. D., Cho, J. Y. N., and Gage, K. S.: Interactive comment on "Comment on "Reinterpreting aircraft measurement in anisotropic scaling turbulence" by Lovejoy et al. (2009)", Atmos. Chem. Phys. Discuss., 9, C9797-C9798, 2010b.

Lindborg, E., Tung, K. K., Nastrom, G. D., Cho, J. Y. N., and Gage, K. S.: Interactive Comment on "Comment on "Reinterpreting aircraft measurements in anisotropic scaling turbulence" by Lovejoy et al. (2009)", Atmos. Chem. Phys. Discuss., 9, C7688-C7696, 2009.

Lovejoy, S. and Schertzer, D.: Space-time cascades and the scaling of ECMWF reanalyses: fluxes and fields, Nonlin. Proc., Geophys., in preparation, 2010a.

Lovejoy, S. and Schertzer, D.: Towards a new synthesis for atmospheric dynamics: space-time cascades, Atmos. Res., 96, 1-52, doi:10.1016/j.atmosres.2010.01.004, 2010b.

Why anisotropic turbulence matters: another reply

S. Lovejoy et al.

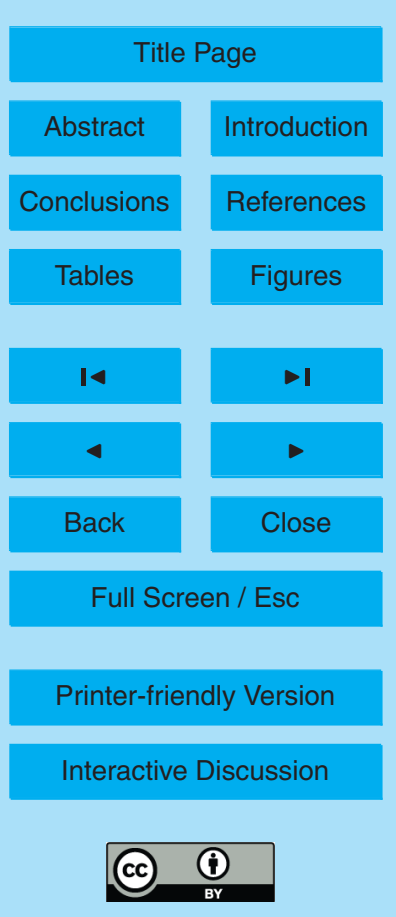


Lovejoy, S., Tuck, A. F., Hovde, S. J., and Schertzer, D.: Is isotropic turbulence relevant in the atmosphere?, Geophys. Res. Lett., L14802, doi:10.1029/2007GL029359, 2007.

Lovejoy, S., Tuck, A. F., Schertzer, D., and Hovde, S. J.: Reinterpreting aircraft measurements in anisotropic scaling turbulence, Atmos. Chem. Phys., 9, 5007-5025, 2009,

5 http://www.atmos-chem-phys.net/9/5007/2009/.

Ngan, K., Straub, D. N., and Bartello, P.: Three-dimensionalisation of freely-decaying twodimensional flows, Phys. Fluids, 16, 2918-2932, 2004.

Schertzer, D.: Interactive comment on "Comment on "Reinterpreting aircraft measurements in anisotropic scaling turbulence" by Lovejoy et al. (2009) by E. Lindborg et al., Atmos. Chem.

$10 \quad$ Phys. Discuss., 9, C8605-C8610, 2009.

Schertzer, D., Lovejoy, S., Schmitt, F., Chigirinskaya, Y., and Marsan, D.: Multifractal cascade dynamics and turbulent intermittency, Fractals, 5, 427-471, 1977.

Smith, K. S.: Comment on: "The $k^{-3}$ and $k^{-5 / 3}$ energy spectrum of atmospheric turbulence: Quasigeostrophic two-level model simulation”, J. Atmos. Sci., 61, 937-941, 2004.

Stolle, J., Lovejoy, S., and Schertzer, D.: The stochastic multiplicative cascade structure of deterministic numerical models of the atmosphere, Nonlin. Processes Geophys., 16, 607621,2009 ,

http://www.nonlin-processes-geophys.net/16/607/2009/.

\section{ACPD}

10, 7495-7506, 2010

Why anisotropic turbulence matters: another reply

S. Lovejoy et al.

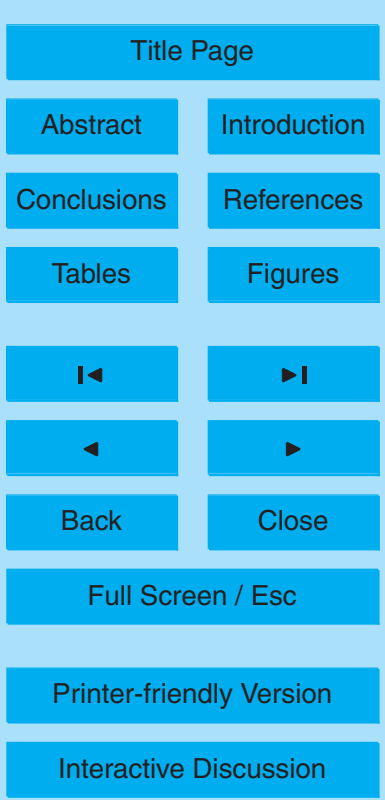




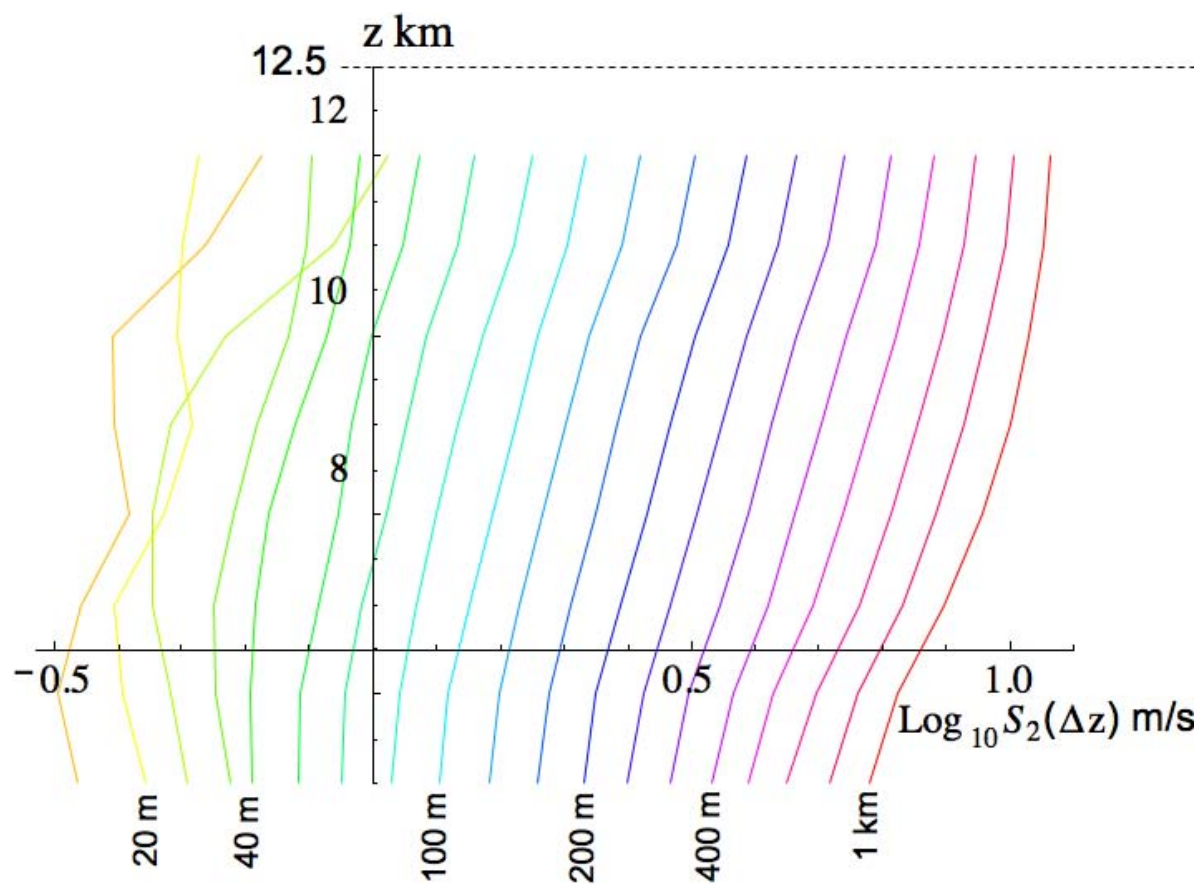

Fig. 1. The altitude as a function of the associated root mean square horizontal wind difference: for 237 drop sondes from the Pacific 2004 experiment (as described in Lovejoy et al., 2007). Each curve is for a different, fixed vertical lag $\Delta z$. Lags increase by factors of $10 \times 0.1=1.25$ from left to right, between $15 \mathrm{~m}$ and $1 \mathrm{~km}$ (some are indicated explicitly). The altitude is the mean over a $1 \mathrm{~km}$ thick layer so that $12 \mathrm{~km}$ indicates an average over a layer from $11.5-12.5 \mathrm{~km}$. The approximate mean aircraft flight level $(12.5 \mathrm{~km})$ is indicated by a dashed line. The near uniform spacing between lines corresponds to a vertical spectrum of $k^{-2.4}$.
10, 7495-7506, 2010

\section{Why anisotropic turbulence matters: another reply}

S. Lovejoy et al.

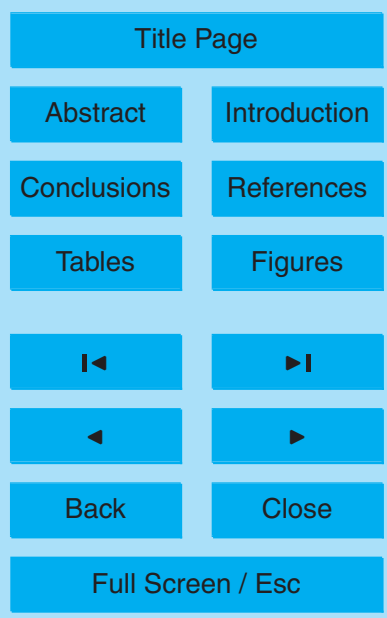

Printer-friendly Version

Interactive Discussion 